\title{
Local time in diffusive media and applications to imaging
}

\author{
Vincent Rossettd* \\ Université de Grenoble I - LPMMC / CNRS, \\ 25 avenue des Martyrs, \\ 38042 Grenoble CEDEX 09, France
}

(Dated: January 11, 2021)

\begin{abstract}
Local time is the measure of how much time a random walk has visited a given position. In multiple scattering media, where waves are diffuse, local time measures the sensitivity of the waves to the local medium's properties. Local variations of absorption, velocity and scattering between two measurements yield variations in the wave field. These variations are proportionnal to the local time of the volume where the change happened and the amplitude of variation. The wave field variations are measured using correlations and can be used as input in a inversion algorithm to produce variation maps. The present article gives the expression of the local time in dimensions one, two and three and an expression of its fluctuations, in order to perform such inversions and estimate their accuracy.
\end{abstract}

\section{INTRODUCTION}

Standard imaging techniques in physical sciences are based on the deterministic nature of wave transport. In transmission, absorption or reflection imaging such as in the different types of optical microscopy, radiography, ultrasonography, the production of an image relies on the fact that the source wavelength is much larger than the irrelevant and unpredictable variations of the properties of the propagation medium. As long as only a small fraction of the energy is scattered by these irregularities, scattering is negligible and the medium is transparent. Media where scattering is not negligible are called scattering media. In scattering media a substancial amount of the wave energy is scattered and more advanced imaging techniques are necessary. Several strategies have been designed to obtain accurate and reliable results. In optical coherence tomography for instance, the fraction of signal that has been scattered more than once is filtered out. However, when scattering is so strong that the largest part of the signal has been multiply scattered, these strategies fail: the medium becomes opaque.

In strongly scattering media, wave propagation reaches rapidly a diffuse regime: the intensity in space and time evolves according to a diffusion equation. Diffusive media, such as fog, are commonly believed to yield fuzzy images. However, it was recently realized that even in the diffuse regime, the measured signals carry informations. The wave recorded at a given position is the superposition of partial waves which trajectories follow a certain statistics determined by the diffusion equation. These partial waves statistically explore the whole medium and are sensitive to its local properties. The measured signal therefore contains informations concerning the transport properties of the medium collected during their propagation, but these informations are entangled in such a way that the raw experimental measurements cannot be directly used to produce a regular image of the medium.

\footnotetext{
* Author's e-mail vincent.rossetto@grenoble.cnrs.fr
}

In heterogeneous, turbid media, techniques based on the correlations have proved efficient to extract some relevant informations. The nowadays commonly used diffusing-wave spectroscopy (DWS) allows one to measure the diffusion of scatterers [1] in diffuse regime for the waves. However, these techniques remain poorly sensitive for imaging a medium with static scatterers. It is nonetheless possible to increase the sensitivity if one considers the field rather than the intensity [2]. The field is the displacement, the pressure or the electric field, depending on the nature of the wave. It can be recorded for waves with frequencies below the order of $\mathrm{THz}$, which are encountered in acoustics, seismology or material sciences. Modern recording devices provide precise amplitude records for them at an appropriate, higher sampling frequency. The wave field records are used to compare two states of the same system at different times and produce an image of the changes in the system, this is why one speaks of differential imaging. The achieved sensitivity is so strong that a single change on one scatterer among thousands of others can be detected. Small relative velocity changes, of the order of $10^{-5}$, have also been measured using a technique based on the diffuse part of acoustic records 3, 4] or the late part of seismograms, the seismic coda [5, 6]. These results have been obtained by measuring the time shifts between waveforms that maximize their correlations. The name of coda wave interferometry (CWI) is often met to gather these approaches. It was recently shown that the maximum of correlation obtained in this way contains informations about the changes in the scattering properties of the medium [7]. The data extracted by correlation are used as input in inversion techniques to produce images. Defects appearing in a concrete structure have been located correctly by an error minimization inversion [8]. Local time has been used as a kernel in seismology to observe the depth sensitivity of the seismic coda to velocity perturbations 9 . In a volcano, precursor structural changes to an eruption have been observed using LOCADIFF [9].

The underlying common principle of CWI and LOCADIFF is based on the observation that the sensitivity 
of the diffuse waves to a change in the medium is proportionnal to the time spent at the position where the change has occured. This time is called the local time. Local time is a way to focus on a position in a heterogeneous medium. It is a random quantity, because of the random nature of the wave propagation in hereogeneous media, but its average value is well defined. However the distribution of local time is defined only in one dimension. To sort out the statistical error in practical applications, one needs to know the order of magnitude of the fluctuations. In the present work, I introduce the notion of local time and compute its average value in diffusive media. To obtain the fluctuations, it is necessary to introduce a resolution length on which the fluctuations depend.

The present article aims at introducing the concept of local time and at giving the possibility to investigate its relevance in every field of Physics where waves are strongly scattered. Parts of this article are technical; they are intended to give a complete material on the subject of local time and its fluctuations. These parts can be skipped on the first reading and are pointed out along the text. The article starts with the introduction of the local time and some of its general properties. A brief mathematical definition is sketched at the end of Section [II The Brownian bridge is introduced elementarily in paragraph [IIA and more mathematically in paragraph IIIB. Section IV briefly presents the computation of the average local time in dimensions one, two and three using the Brownian bridge properties. Only the paragraph IVA is technical, the other paragraph of Section IV being dedicated to giving the result and commenting on their properties. The distribution of local time is determined in the Section $\mathrm{V}$ using the technique presented in paragraph $\mathrm{VB}$. The distribution in dimension one is given in $\mathrm{VC}$. To obtain regular distributions in dimensions higher than one, it is necessary to introduce a small length scale corresponding to the resolution. I compute the fluctuations of the local time in the other paragraph of Section $\mathrm{V}$ and show that it depends on the resolution for dimensions higher than one. The applications of these results in experiments are discussed in Section VI. Possible improvements are evoked in the conclusion. The reader interested only in the results for applications may focus on the text and the results of equations (16), (18), (20), (29), (32) and (34) and skip the technical paragraphs IIIB, IVA, VB.

\section{PROPERTIES OF THE LOCAL TIME}

Let us consider a disordered medium in which particles or waves travel following random trajectories. The distribution of probability for the particles' random trajectories coincides with the energy statistics of partial waves. The particles or the waves are emitted from the origin at time $t_{0}$ and are scattered by the heterogeneities of the medium. The source term is therefore a Dirac delta function in space and time. At time $t$, the probability to find a particle - or the fraction of wave energy in an elementary volume $\mathrm{d}^{d} \boldsymbol{x}$ centered at the position $\boldsymbol{x}$ is noted $G(\boldsymbol{x}, t) \mathrm{d}^{d} \boldsymbol{x} . G(\boldsymbol{x}, t)$ is the elementary solution - or Green's function - of the transport equation, a partial differential equation such as the diffusion equation or the radiative transfer equation [10].

A detection device at the position $\boldsymbol{R}$ is sensitive only to $G(\boldsymbol{R}, t)$ where $\boldsymbol{R}$ is in the close neighborhood of $\boldsymbol{R}$ such that at a given time $t$, it only detects the signal sent from the origin reaching $\boldsymbol{R}$ at this time. The signal recorded in $\boldsymbol{R}$ is thus the Green's function, or impulse response, from the origin to $\boldsymbol{R}$. During time $t$, the observed signal is a superimposition of signals due to random trajectories that have explored the medium. As an example, consider photons emitted in a pulse at time $t=0$ from a source located at the origin. A photo-detector located in $\boldsymbol{R}$ measures the intensity $I(t)$ along time. We suppose that the fraction of photons absorbed by the photodetector represent a negligible fraction of the measured intensity and does not affect the experiment. Suppose that after this measurement, the absorption $\mu(\boldsymbol{x})$ changes non-uniformly by the quantity $\Delta \mu(\boldsymbol{x})$. We now denote by $I^{\prime}(t)$ the intensity measured in $\boldsymbol{R}$ after these changes if the source sends a rigorously identical pulse. If photons spend a time $\delta t$ at the position $\boldsymbol{x}$, their contribution to the logarithm of the intensity will be reduced by $\Delta \mu(\boldsymbol{x}) \delta t$. The average local time $L(\boldsymbol{x}, t) \mathrm{d}^{d} \boldsymbol{x}$ is the time spent on average by the photons in the elementary volume $\mathrm{d}^{d} \boldsymbol{x}$ around $\boldsymbol{x}$ during the whole propagation time $t$ after the pulse emission. The logarithm of the intensity is therefore reduced by $\Delta \mu(\boldsymbol{x}) L(\boldsymbol{x}, t) \mathrm{d}^{d} \boldsymbol{x}$. As a conclusion the total observed logarithm of intensity will be modified by

$$
\log \frac{I^{\prime}(t)}{I(t)}=-\iiint \Delta \mu(\boldsymbol{x}) L(\boldsymbol{x}, t) \mathrm{d}^{d} \boldsymbol{x} .
$$

The observed intensity is therefore modified by the average local time. Supposing that we had not only a source and a receiver but many of each, an imaging process would consist in measuring the variation of intensity between each source and each receiver and performing a mathematical inversion to reconstruct the value of $\Delta \mu(\boldsymbol{x})$. In this article, I compute the expressions of $L$ in diffusive media and its fluctuations. By symmetry considerations, it is easy to see that these quantities only depend on the distances $R=\|\boldsymbol{R}\|, a=\|\boldsymbol{x}\|$ and $b=\|\boldsymbol{x}-\boldsymbol{R}\|$ at position $\boldsymbol{x}$ (see the figure1).

Let us now mathematically define the local time. Consider a function $f$ defined on $\mathbf{R}^{d}$ and a random continuous process $\boldsymbol{X}_{u}$ for $0 \leq u \leq t$, for instance a propagation process. If the stochastic average of $\int_{0}^{t} f\left(\boldsymbol{X}_{u}\right) \mathrm{d} u$ over all realisations of $\boldsymbol{X}$ is expressed as a space integral of $f$ weighted by a function $L_{d}(\boldsymbol{x}, t)$,

$$
\left\langle\int_{0}^{t} f\left(\boldsymbol{X}_{u}\right) \mathrm{d} u\right\rangle=\int_{\mathbf{R}^{d}} f(\boldsymbol{x}) L_{d}(\boldsymbol{x}, t) \mathrm{d}^{d} \boldsymbol{x},
$$

then $L_{d}(\boldsymbol{x}, t)$ is called the average local time of the process $\boldsymbol{X}$. The brackets $\langle\cdot\rangle$ denote the stochastic average 


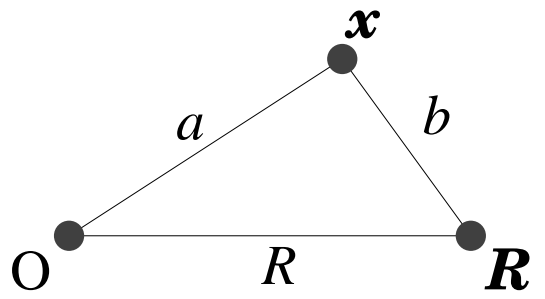

FIG. 1. Coordinates used in the article. $\boldsymbol{R}$ is a fixed point at distance $R=\|\boldsymbol{R}\|$ from the origin. The generic point $\boldsymbol{x}$ is located at the distances $a=\|\boldsymbol{x}\|$ from the origin and $b=$ $\|\boldsymbol{x}-\boldsymbol{R}\|$ from $\boldsymbol{R}$ respectively (dimension $d \geqslant 2$ ).

over the realisations of $\boldsymbol{X}$. Equation (2) is sometimes called the occupation time formula [11]. Such a formula was originally imagined by Trotter [12], inspired by Lévy's "mesure de voisinage" [13]. The usefulness of this formula is to transform a stochastic average into a regular integral. The average local time is a positive, continuous function of space. Using the definition (2) with the constant function $f(\boldsymbol{x})=1$, we find

$$
\int_{\mathbf{R}^{d}} L_{d}(\boldsymbol{x}, t) \mathrm{d}^{d} \boldsymbol{x}=t .
$$

This relation expresses the intuitive fact that the particle spends in total the time $t$ in the medium and that the average local density of this time is distributed according to the function $\boldsymbol{x} \mapsto L_{d}(\boldsymbol{x}, t)$. In other words, Equation (3) translates into mathematics the physical notion of average local time. If we use now the definition (2) with the function $f(\boldsymbol{y})=\delta^{d}(\boldsymbol{x}-\boldsymbol{y})$ we find the expression of the average local time as a stochastic average

$$
L_{d}(\boldsymbol{x}, t)=\left\langle\int_{0}^{t} \delta^{d}\left(\boldsymbol{X}_{u}-\boldsymbol{x}\right) \mathrm{d} u\right\rangle .
$$

The expressions (2), (3) and (4) do not depend on the stochastic process $\boldsymbol{X}_{t}$. They apply not only to Brownian motion, but also for instance to the solutions of the radiative transfer in a disordered medium [14-16]. The local time of Brownian motion was studied mostly in Mathematics: The average local time has been explicitely computed in the case of a one-dimensional reflecting Brownian motion by Gittenberger and Louchard [17] and the probability distribution of local time in one dimension has been also determined by Pitman [18]. Other presentations of the local time of Brownian motion can be found in Ref. [11].

\section{THE BROWNIAN BRIDGE}

\section{A. Definition of the Brownian bridge}

Let us consider a medium where the intensity is diffuse $\frac{\partial I}{\partial t}(\boldsymbol{x}, t)=D \nabla^{2} I(\boldsymbol{x}, t) . D$ is the diffusion constant and fully characterizes the transport in the medium.
We consider a point source located at the origin of the coordinate system. A receiver is located at point $\boldsymbol{R}$. The distance of a point $\boldsymbol{x}$ to the origin and to $\boldsymbol{R}$ are denoted respectively $a$ and $b$ (see Figure 1).

In an infinite space of dimension $d$ the diffusion kernel, also called elementary solution or Green's function, at distance $r$ and time $t$ is given by the Gaussian distribution

$$
G_{d}(r, t)=\frac{1}{(4 \pi D t)^{d / 2}} \exp \left[-\frac{r^{2}}{4 D t}\right] .
$$

This distribution is equal to the probability distribution function for the position of the point $\boldsymbol{B}_{t}$ of a Brownian motion starting at the origin at time $t=0$. For a given position $\boldsymbol{R}$ and a given time $t$, the Brownian bridge $\boldsymbol{P}^{\boldsymbol{R}, t}$ is defined as the stochastic process

$$
\boldsymbol{P}_{u}^{\boldsymbol{R}, t}=\boldsymbol{B}_{u}+\frac{u}{t}\left(\boldsymbol{R}-\boldsymbol{B}_{t}\right),
$$

where $0 \leq u \leq t$ is the time variable. The second term of the right-hand side of (6) "forces" the process to go to $\boldsymbol{R}$ at time $t$. The Brownian bridge $\boldsymbol{P}^{\boldsymbol{R}, t}$ selects all the Brownian trajectories satisfying the boundary conditions $\boldsymbol{P}_{0}^{\boldsymbol{R}, t}=0, \boldsymbol{P}_{t}^{\boldsymbol{R}, t}=\boldsymbol{R}$. When computing the local time using the definition (4), the statistical average is performed over all the realizations of the Brownian bridge $\boldsymbol{P}^{\boldsymbol{R}, t}$. We prove this statement in the next, more technical paragraph, along with other mathematical properties.

\section{B. Characteristic function of the drifted Brownian bridge}

The Brownian bridge (6) is a Gaussian stochastic process of mean $\frac{u}{t} \boldsymbol{R}$. The covariance of the Brownian motion $\boldsymbol{B}_{u}$ is $\left\langle\boldsymbol{B}_{u} \cdot \boldsymbol{B}_{v}\right\rangle=2 D \min (u, v)$, the covariance of the Brownian bridge with $\boldsymbol{R}=0$ is therefore

$$
\left\langle\boldsymbol{P}_{u}^{0, t} \cdot \boldsymbol{P}_{v}^{0, t}\right\rangle=\frac{2 D}{t} u(t-v), \quad(u \leq v) .
$$

As it is a Gaussian process, its characteristic function is

$$
\phi_{0}(\mathbf{q}, u)=\left\langle\exp \mathbf{i q} \cdot \boldsymbol{P}_{u}^{0, t}\right\rangle=\exp \left[-\frac{D}{t} u(t-u) \mathbf{q}^{2}\right] .
$$

The characteristic function for $\boldsymbol{R} \neq 0$ is obtained from Eq. (8) using a drift

$$
\phi_{\boldsymbol{R}}(\mathbf{q}, u)=\exp \left[\mathrm{i} \frac{u}{t} \mathbf{q} \cdot \boldsymbol{R}-\frac{u(t-u)}{t} D \mathbf{q}^{2}\right] .
$$

The usual properties of the characteristic function state that for an arbitrary function $f$, of $d$-dimensional Fourier transform $\tilde{f}$, we have

$$
\left\langle f\left(\boldsymbol{P}_{u}^{\boldsymbol{R}, t}\right)\right\rangle=\int \frac{\mathrm{d}^{d} \mathbf{q}}{(2 \pi)^{d}} \phi_{\boldsymbol{R}}(\mathbf{q}, u) \tilde{f}(\mathbf{q}) .
$$


Summing the characteristic functions of $\boldsymbol{P}^{\boldsymbol{R}, t}$ over $\boldsymbol{R}$ with the Gaussian weight $G_{d}(\|\boldsymbol{R}\|, t)$, we obtain the characteristic function of the Brownian motion:

$$
\int_{\mathbf{R}^{d}} G_{d}(\|\boldsymbol{R}\|, t) \phi_{\boldsymbol{R}}(\mathbf{q}, u) \mathrm{d}^{d} \boldsymbol{R}=\tilde{G}_{d}(\mathbf{q}, u),
$$

which expresses the equivalence between Brownian motion and a collection of Brownian bridges. The Brownian bridge $\boldsymbol{P}^{\boldsymbol{R}, t}$ selects the Brownian motion trajectories for which $\boldsymbol{B}_{t}=\boldsymbol{R}$.

\section{AVERAGE LOCAL TIME FOR DIFFUSION}

\section{A. General expression of the average local time}

The mathematics for the computation of the local time are detailed in this paragraph. The results are given in the following paragraphs of this Section. I use as in Section III the notations $a=\|\boldsymbol{x}-A\|, b=\|\boldsymbol{x}-B\|$ and $R=\|B-A\|$. To compute the average local time $L_{d}(\boldsymbol{x}, t)$ from the equation (41) let us use the characteristic function formula (10) with the function $f(\boldsymbol{y})=\delta^{d}(\boldsymbol{x}-\boldsymbol{y})$ :

$$
L_{d}(\boldsymbol{x}, t)=\int_{0}^{t} \mathrm{~d} u \int_{\mathbf{R}^{d}} \frac{\mathrm{d}^{d} \mathbf{q}}{(2 \pi)^{d}} \phi_{\boldsymbol{R}}(\mathbf{q}, u) \mathrm{e}^{-\mathrm{iq} \cdot \boldsymbol{x}} .
$$

The result is obtained by inserting the expression (9) and performing the inverse Fourier transform. We get

$$
L_{d}(\boldsymbol{x}, t)=\frac{t^{d / 2} \mathrm{e}^{\frac{\boldsymbol{R}^{2}}{4 D t}}}{(4 \pi D)^{d / 2}} \int_{0}^{t} \frac{\mathrm{e}^{-\frac{a^{2}}{4 D u}}}{u^{d / 2}} \frac{\mathrm{e}^{-\frac{b^{2}}{4 D(t-u)}}}{(t-u)^{d / 2}} \mathrm{~d} u .
$$

Let us relate this result to the solution of the diffusion equation (5)

$$
L_{d}(\boldsymbol{x}, t)=\frac{1}{G_{d}(R, t)} \int_{0}^{t} G_{d}(a, u) G_{d}(b, t-u) \mathrm{d} u .
$$

The latter formula has a simple interpretation: the integrand is the probability of going from $A$ to $\boldsymbol{x}$ in time $u$ and then from $\boldsymbol{x}$ to $B$ in time $t-u$. This probability is integrated over $u$ since the point $\boldsymbol{x}$ can be visited at any moment. The result is divided, as required by the Bayes formula, by the probability of going from $A$ to $B$ in time $t$, the boundary conditions. This formula is valid for any transport function $G_{d}$ in multiple scattering media [7]. As diffusion is a Markov process, equality (3) follows from the Chapman-Kolmogorov relation [19]

$$
\int_{\mathbf{R}^{d}} G_{d}(a, u) G_{d}(b, t-u) \mathrm{d}^{d} \boldsymbol{x}=G_{d}(R, t) .
$$

To compute the time convolution in Eq. (12), I will use the Laplace transform in time. Noting $\hat{f}_{j}(s)$ the Laplace transform of a function $f_{j}(t)$, the Laplace transform of

$$
\int_{0}^{t} f_{1}\left(u_{1}\right) \mathrm{d} u_{1} \cdots \int_{0}^{t} f_{n}\left(u_{n}\right) \delta\left(t-u_{1}-\cdots-u_{n}\right) \mathrm{d} u_{n}
$$

as a function of $t$ is equal to $\hat{f}_{1}(s) \cdots \hat{f}_{n}(s)$ (see Appendix $\mathrm{A}$ ). The Laplace transforms $\hat{G}_{d}(a, s)$ for $d=1,2$ and 3 are given in the table I In the Laplace domain, I include the weighting term $G_{d}(R, t)^{-1}$ of (12) by defining

$$
\bar{f}(s)=\int_{0}^{\infty} \mathrm{e}^{-s t} f(t) G_{d}(R, t) \mathrm{d} t
$$

to express Equation (12) undeg the simple form

$$
\overline{L_{d}}(\boldsymbol{x}, s)=\hat{G}_{d}(a, s) \hat{G}_{d}(b, s) .
$$

The function $\hat{G}_{d}(a, s)$ is singular in $a=0$. The type of

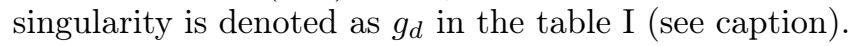

\begin{tabular}{|c|c|l||c|}
\hline$d$ & $\hat{G}_{d}(\|\boldsymbol{x}\|, s)$ & Reference & Singularity $g_{d}(\boldsymbol{x})$ \\
\hline 1 & $\frac{1}{\sqrt{4 D s}} \exp \left[-|x| \sqrt{\frac{s}{D}}\right]$ & 29.3 .84 & $\frac{1}{2}|x|$ \\
\hline 2 & $\frac{1}{2 \pi D} K_{0}\left(\|\boldsymbol{x}\| \sqrt{\frac{s}{D}}\right)$ & 29.3 .120 & $\frac{1}{2 \pi} \ln \|\boldsymbol{x}\|$ \\
\hline 3 & $\frac{1}{4 \pi D\|\boldsymbol{x}\|} \exp \left[-\|\boldsymbol{x}\| \sqrt{\frac{s}{D}}\right]$ & 29.3 .82 & $-\frac{1}{4 \pi\|\boldsymbol{x}\|}$ \\
\hline
\end{tabular}

TABLE I. Table of Laplace transforms in time of the diffusion kernel (5) for the dimensions 1, 2 and 3. The numbers refer to the equation in the handbook by Abramowitz and Stegun [20]. When $\boldsymbol{x} \rightarrow 0, \hat{G}_{d}(\|\boldsymbol{x}\|, s)$ is equivalent to $g_{d}(\boldsymbol{x}) / D$ where the singularity $g_{d}(\boldsymbol{x})$ is the Green's function of the Laplace equation in $d$ dimensions.

\section{B. Average local time in one dimension}

To obtain the expression of the average local time in one dimensions $(d=1)$, we compute the inverse Laplace transform of

$$
G_{1}(a, s) G_{1}(b, s)=\frac{1}{4 D s} \exp \left[-(a+b) \sqrt{\frac{s}{D}}\right],
$$

which is, according to the reference [20, eq. 29.3.83], a complementary error function. Introducing the notation $\operatorname{erfc}(x)=2 / \sqrt{\pi} \int_{x}^{\infty} \exp \left[-u^{2}\right] \mathrm{d} u$, we have

$$
L_{1}(x, t)=\sqrt{\frac{\pi t}{4 D}} \operatorname{erfc}\left[\frac{a+b}{\sqrt{4 D t}}\right] \exp \left[\frac{R^{2}}{4 D t}\right] .
$$

A particularity in expression (16) is that for all $x$ between $A$ and $B, L_{1}$ has the same value because $a+b=R$ then ; This is a consequence of the Markovian character of the diffusion kernel: as long as the process is between $A$ and $B$, it has an equal probability to visit any position. Let us also remark that the average local time $L_{1}$ remains finite in $A$ and $B$, but that is these points, there is a slope change, as for $g_{1}(x)$.

When $A$ and $B$ coincide, we have $R=0$ and $a=b$. In this case, we note the local time $\ell_{d}(a, t)$. In dimension one we have therefore $\ell_{1}(a, t)=\sqrt{\pi t / 4 D} \operatorname{erfc}(a / \sqrt{D t})$. 


\section{Average local time in two dimensions}

To compute the exact result in two dimensions $(d=$ 2 ), let us use the following result, demonstrated in the appendix B

$$
\int_{0}^{1} \frac{\mathrm{e}^{-\alpha / u-\beta /(1-u)} \mathrm{d} u}{u(1-u)}=2 \mathrm{e}^{-\alpha-\beta} K_{0}(2 \sqrt{\alpha \beta}) .
$$

$K_{0}$ is the modified Bessel function of the second kind and of order 0 . Replacing $\alpha$ by $a^{2} / 4 D t$ and $\beta$ by $b^{2} / 4 D t$ and introducing the result of (17) in the Formula (12) we obtain the local time kernel in dimension 2

$$
L_{2}(\boldsymbol{x}, t)=\frac{1}{2 \pi D} \exp \left[\frac{R^{2}-a^{2}-b^{2}}{4 D t}\right] K_{0}\left(\frac{a b}{2 D t}\right) .
$$

Remark that the average local time $L_{2}$ has a logarithmic divergence in $A$ and $B$, like $g_{2}(\boldsymbol{x})$ at the origin. When $A$ and $B$ coincide, the local time is expressed by $\ell_{2}(a, t)=$ $\frac{1}{2 \pi D} \exp \left[-\frac{a^{2}}{2 D t}\right] K_{0}\left(\frac{a^{2}}{2 D t}\right)$ as already shown by Pacheco and Snieder [5].

\section{Average local time in three dimensions}

In three dimensions $(d=3)$, the same method as in one dimension is used. Remarkably the product

$$
\hat{G}_{3}(a, s) \hat{G}_{3}(b, s)=\frac{\exp \left[-(a+b) \sqrt{\frac{s}{D}}\right]}{(4 \pi D)^{2} a b}
$$

is equal to $\hat{G}_{3}(a+b, s)$ multiplied by a constant. The inversion is straightforward and yields

$$
L_{3}(\boldsymbol{x}, t)=\frac{a+b}{4 \pi D a b} \exp \left[\frac{R^{2}-(a+b)^{2}}{4 D t}\right] .
$$

In this expression the time behaviour is exclusively contained in the exponential. Note that the argument of the exponential is negative thanks to the triangular inequality $a+b \geqslant R$ for all $\boldsymbol{x}$. Remark also that the average local time in three dimensions diverges in $A$ as $a^{-1}$ and in $B$ as $b^{-1}$ like $g_{3}(\boldsymbol{x})$ at the origin. For coincident $A$ and $B$ we have $\ell_{3}(a, t)=\frac{1}{2 \pi D a} \exp \left[-\frac{a^{2}}{D t}\right]$ which was also given by Pacheco and Snieder [5].

\section{FLUCTUATIONS OF LOCAL TIME FOR DIFFUSION}

\section{A. The origin of the fluctuations of local time}

If one considers single Brownian trajectories separately, the local time in $\boldsymbol{x}$ depends on the particular realization of the Brownian motion. Local time is therefore a random quantity and the origin of its randomness is the different realizations of Brownian motion. However, in a perfect diffusive media each trajectory is realized by a partial wave which carries an energy proportional to its probability. In such a perfect medium, the local time would always be equal to its average value. Yet, actual diffusive media are not perfect since they have a correlation length, called the mean free path. As a consequence, some Brownian trajectories are not followed by any partial waves. Different realizations of the medium (sometimes referred to as realizations of the medium's disorder) select and exclude different Brownian trajectories. Only a sample of all Brownian trajectories is realized in a given medium, the value of the local time therefore differs from the results of the previous Section and the difference depends on the positions of all the constituents of the material. Local time is therefore a random variable in wave propagating media as well, but its randomness roots in the different realizations of disorder. The sample space is made of the configurations of the medium, and not of the realizations of a stochastic process. Ergodicity states that these sample spaces lead to the same statistics. In this section, I compute the probability distribution function of the local time of a Brownian bridge and thanks to the ergodic equivalence, use it to compute the fluctuations of the local time with respect to the medium's disorder.

\section{B. Moments of the local time distribution}

In this paragraph, we perform the computation of the moments of the local time distribution. The results are presented and discussed in the following, less technical, paragraphs. We start from the definition of the moment of order $m$ of the local time distribution in dimension $d$

$$
\mu_{d}^{m}(\boldsymbol{x}, t)=\left\langle\left(\int_{0}^{t} \delta^{(d)}\left(\boldsymbol{P}_{u}^{\boldsymbol{R}, t}-\boldsymbol{x}\right) \mathrm{d} u\right)^{m}\right\rangle .
$$

When $m=0$, the moment is $\mu_{d}^{0}=1$. In the following developments, we will assume $m \geq 1$. Let us expand every Dirac $\delta$-function as a Fourier integral

$$
\mu_{d}^{m}(\boldsymbol{x}, t)=\int \prod_{j=1}^{m} \mathrm{~d} u_{j} \prod_{j=1}^{m} \frac{\mathrm{d}^{d} \mathbf{q}_{j}}{(2 \pi)^{d}}\left\langle\mathrm{e}^{\mathrm{i} \sum_{j=1}^{m} \mathbf{q}_{j} \cdot\left(\boldsymbol{P}_{u_{j}, t}^{\boldsymbol{R}, \boldsymbol{x}}\right)}\right\rangle .
$$

Let us reorder the times such that $u_{0}=0 \leq u_{1} \leq \cdots \leq$ $u_{m} \leq u_{m+1}=t$. The brackets in the above equation contain the characteristic function of a Brownian bridge visiting $\boldsymbol{x}$ at times $u_{1} \leq \ldots, \leq u_{m}$. Therefore it is a sequence of Brownian bridges, the first between $\left(A, u_{0}\right)$ and $\left(\boldsymbol{x}, u_{1}\right)$, then $m-1$ between $\left(\boldsymbol{x}, u_{i}\right)$ and $\left(\boldsymbol{x}, u_{i+1}\right)$ for $1 \leq i \leq m-1$ and the last one between $\left(\boldsymbol{x}, u_{m}\right)$ and $\left(B, u_{m+1}\right)$. The characteristic function is thus Gaussian with correlation matrix $C_{i j}=2 D u_{i}\left(t-u_{j}\right) / t$, according to the equation (7). The inverse Fourier transform leads to an expression with the same structure as 
the Equation (11), noting $a_{1}=a, a_{m+1}=b$ and $a_{i}=0$ for $2 \leq i \leq m$,

$$
\mu_{d}^{m}(\boldsymbol{x}, t)=\frac{m !}{G_{3}(R, t)} \int \prod_{i=1}^{m} \mathrm{~d} u_{i} \prod_{i=1}^{m} G_{d}\left(a_{i}, u_{i+1}-u_{i}\right) .
$$

The integral is performed for the values of $u_{i}$ obeying $0=u_{0} \leq u_{1} \leq \cdots \leq u_{m+1}=t$. Let us change the variables $u_{i}$ into $v_{i}=u_{i+1}-u_{i}$ such that the condition on times is now $\sum_{0}^{m} v_{i}=t$. In the Appendix A, I show that the Laplace transform of this integral is a product of Laplace transforms

$$
\overline{\mu_{d}^{m}}(\boldsymbol{x}, s)=m ! \hat{G}_{d}(a, s) \hat{G}_{d}(b, s) \hat{G}_{d}(0, s)^{m-1} .
$$

In the case $m=1$ we retrieve the expression for the local time (12) $\left(\mu_{d}^{1}=L_{d}\right)$. The moments $\overline{\mu_{d}^{m}}(\boldsymbol{x}, s)$ depend on the order $m$ like $m ! \alpha^{m}$, for some number $\alpha$, which is characteristic for an exponential distribution of width $\alpha$. Denoting by $\tau$ the value of the local time, we deduce that its probability distribution is

$$
\begin{aligned}
\overline{p_{d}}(\boldsymbol{x}, s ; \tau) & =\overline{S_{d}}(\boldsymbol{x}, s) \delta(\tau)+\frac{\overline{L_{d}}(\boldsymbol{x}, s)}{\hat{G}_{d}(0, s)^{2}} \mathrm{e}^{-\frac{\tau}{\hat{G}_{d}(0, s)}}, \\
\overline{S_{d}}(\boldsymbol{x}, s) & =\hat{G}_{d}(R, s)-\frac{\overline{L_{d}}(\boldsymbol{x}, s)}{\hat{G}_{d}(0, s)} .
\end{aligned}
$$

Observe that the distribution of local time displays a singularity at $\tau=0$ which expresses the fact that some trajectories do not visit the neighbourhood of $\boldsymbol{x}$. The term $\bar{S}_{d} \delta(\tau)$ ensures the normalization of the probability distribution function. I call this term the singular term and the second one the regular term of the probability distribution function, denote it by $\left\{p_{d}(\tau)\right\}$.

\section{Distribution of local time in one dimension}

In dimension $d=1$, the expression (23) for $\overline{p_{1}}$ gives for the regular part of the local time distribution

$$
\left\{\overline{p_{1}}(x, s ; \tau)\right\}=\exp \left[-(a+b+2 D \tau) \sqrt{\frac{s}{D}}\right] .
$$

It is of the same form as $\hat{G}_{3}$ so that the regular local time probability distribution function in one dimension

$$
\left\{p_{1}(x, t ; \tau)\right\}=\frac{a+b+2 D \tau}{t} \exp \left(\frac{R^{2}-(a+b+2 D \tau)^{2}}{4 D t}\right) .
$$

This result was first demonstrated by Pitman [18]. The singular part is

$$
\bar{S}_{1}(x, s)=\hat{G}_{1}(R, s)-\frac{\exp \left[-(a+b) \sqrt{\frac{s}{D}}\right]}{\sqrt{4 D s}}
$$

and is therefore a difference of two functions $\hat{G}_{1}$. We obtain

$$
S_{1}(x, t)=1-\exp \left(\frac{R^{2}-(a+b)^{2}}{4 D t}\right) .
$$

$S_{1}(x, t)$ is the probability not to visit the position $x$. Observe that for $x$ located between $A$ and $B$, this probability is equal to 0 because one must visit it to cross from $A$ to $B$.

Writing $\Phi(x)=\sqrt{\pi} \exp \left[x^{2}\right] \operatorname{erfc}(x)$ and $y=(a+$ $b) / \sqrt{4 D t}$, we find the expression for the relative fluctuations of local time kernel in one dimension

$$
\frac{\Lambda_{1}(x, t)}{L_{1}(x, t)}=\left(\frac{4}{\Phi(y)^{2}}-\frac{4 y}{\Phi(y)}-1\right)^{1 / 2} .
$$

The relative fluctuations as a function of $y$ are rather large, even for long times, the minimal value is $\sqrt{4 / \pi-1} \simeq 0.52272$ when $y=0$.

\section{Fluctuations of local time in two dimensions}

In two dimensions $(d=2)$, the expression (22) must be handled with care, because the expression of $\hat{G}_{2}(a, s)$ has a logarithmic divergence when $a \rightarrow 0$. The inverse Laplace transform of the expression (23) does not exist. To regularize this divergence, let us introduce a small length $\varepsilon$ and replace $\hat{G}_{2}(0, s)$ by $\hat{G}_{2}(\varepsilon, s)$. The fluctuations are obtained from the integral

$$
\int_{0}^{t} \mathrm{~d} u \int_{0}^{t-u} \mathrm{~d} v G_{2}(\varepsilon, u) G_{2}(a, v) G_{2}(b, t-u-v)
$$

that I compute in the Appendix $\mathrm{C}$ The divergence in the small distance $\varepsilon$ is logarithmic and we obtain

$$
\mu_{2}^{2}(\boldsymbol{x}, t) \simeq-\frac{L_{2}(\boldsymbol{x}, t)}{2 \pi D} \ln \frac{\varepsilon^{2}}{4 D t}
$$

and the relative fluctuations are approximated by

$$
\frac{\Lambda_{2}(\boldsymbol{x}, t)}{L_{2}(\boldsymbol{x}, t)} \simeq\left(\frac{-\ln \frac{\varepsilon^{2}}{4 D t}}{2 \pi D L_{2}(\boldsymbol{x}, t)}\right)^{1 / 2} .
$$

Let us remark that the fluctuations diverge for small resolution lengths with the same behaviour as the square root of the average local time for small distances from the source or the receiver.

The regularization length $\varepsilon$ is necessary because in dimensions higher than two, Brownian motion visits a given point with probability 0 . This is observed in the fact that $S \rightarrow 1$ for $\varepsilon \rightarrow 0$. To ensure that the measured local time is not identically zero, it is necessary to introduce $\varepsilon$, the radius of a small ball centered at $\boldsymbol{x}$ in which the local time is computed. Therefore, $\varepsilon$ is the resolution length. The average local time remains finite in the limit $\varepsilon \rightarrow 0$ by construction, but the moments of higher orders do not.

\section{E. Distribution of local time in three dimensions}

The local time distribution in three dimensions requires, as in the two dimensional case, a regularization, 
because $G_{3}(a, s)$ diverges when $a \rightarrow 0$. After regularization we obtain $\mu_{3}^{m} \simeq L_{3} m !(4 \pi D \varepsilon)^{m-1}$ and therefore we get an exponential distribution for all $\varepsilon>0$. Writing $\lambda=4 \pi D \varepsilon$, we have

$$
p_{3}(\boldsymbol{x}, t ; \tau)=\left(1-\lambda L_{3}\right) \delta(\tau)+\lambda^{2} L_{3} \exp (-\lambda \tau) .
$$

From the equation (33) we obtain the fluctuations of the measured local time in three dimensions. The result

$$
\frac{\Lambda_{3}(\boldsymbol{x}, t)}{L_{3}(\boldsymbol{x}, t)}=\left(\frac{1}{2 \pi D \varepsilon L_{3}(\boldsymbol{x}, t)}-1\right)^{1 / 2}
$$

states that the fluctuations of the local time in three dimensions are large and grows as the resolution length decreases. Notice that, as in two dimensions, the fluctuations diverge for small resolution lengths with the same behaviour as the square root of the average local time close to the source or the receiver.

\section{APPLICATIONS OF LOCAL TIME TO IMAGING}

In disordered media, the average local time is a measure of the sensitivity of the wave field to a change. The average local time therefore provides the solution to the direct problem: How do the changes in the medium modify the wave fields ? In imaging, however, the changes are unknown and the waves are sent into the medium to monitor them. Thanks to the expressions of the average local time (16), (18) and (20), one can retrieve informations about the changes from the measurement of wave fields of intensity: this is the inverse problem. To perform such inversions, it is necessary to obtain several independant measurements of the systems by installing several sources or receivers. A sketch of an experimental setup is shown in Figure 2. Each pair formed by a source and a receiver provides a measurement of an average local quantity weighted by the local time. Because local times for each pair are different at a given point the inversion is possible. The images produced by such inversions are maps in one, two or three dimensions of the medium. Such inversions have been already performed to image the changes of velocity (CWI) [21] or scattering (LOCADIFF) [8]. Note that only the intensity is required to produce an image of the absorption variation (AV), in which case the average local time imaging technique extends to optics.

In disordered media, the detection and location of strong changes is not a challenge any more: using large wavelength allows using standard imaging techniques. The local time is therefore useful in the case where the changes occurring in the medium are of small amplitude. In such situations, the effects of two separate changes add up, as it was shown for AV in the introduction because the expression (11) is linear in $\Delta \mu(\boldsymbol{x})$. In the case of CWI, time delays add up as long as $\delta v / c \ll 1$, where $\delta v$ is the velocity change and $c$ the average velocity. In LOCADIFF, the losses of correlation due to distant scatterers add

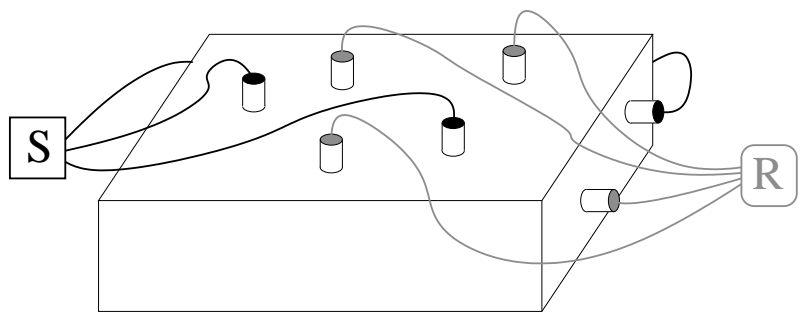

FIG. 2. Sketch of an experimental setup with three sources ( $\mathrm{S}$, black) and four receivers ( $\mathrm{R}$, grey) creating $n=12$ pairs. The source and the receiver must be synchronized. The receivers can be used simultaneously, but the sources should be activated one at a time. If the devices of a pair are too distant, there may be no usable signal. In such case, the pair can be removed from the input vector $M$ and the kernel $K$.

up as long as $c \Delta \sigma L(\boldsymbol{x}, t) \ll 1$, where $\Delta \sigma$ is the change of scattering cross-section [7]. In these three situations, one obtains a linear relation such as Equation (1).

The linear relation is the same in the case of $\mathrm{AV}$, CWI and LOCADIFF. The nature of the input and output physical quantities in different techniques is sum up in the Table II In a practice the medium is divided into a large number $N$ of elements, called voxels, centered at $\boldsymbol{x}_{j}$ $(1 \leq j \leq N)$ of volume $\delta V_{j}$. The medium is equipped with $n$ source-receiver pairs. I denote by $\mathbf{S}_{i}$ and $\mathbf{R}_{i}$ the positions of the source and the receiver of the pair $i$ respectively. The "input" vector $M$ is made of $n$ independent measurements and the output image is a vector $Q$ of size $N$. The relation between the measurements $M$ and the image $Q$ is a matrix equation

$$
K Q=M
$$

in which $K$ is a $n \times N$ matrix called the kernel. The element $K_{i j}$ of the kernel is obtained using $a=\left\|\boldsymbol{x}_{j}-\mathbf{S}_{i}\right\|$, $b=\left\|\boldsymbol{x}_{j}-\mathbf{R}_{i}\right\|$ and $R=\left\|\mathbf{R}_{i}-\mathbf{S}_{i}\right\|$ in the appropriate average local time formula: $K_{i j}=L\left(\mathbf{S}_{i}, \boldsymbol{x}_{j}, \mathbf{R}_{i}, t\right) \delta V_{j}$. In principle, one should install as many source-receiver pairs as the number of voxels of the images to ensure that equation (35) has a solution. However, inversion techniques such as the error-minimization algorithm of TarantolaValette [22], linear minimization or compressive sensing techniques [23] provide in general satisfying results from incomplete informations when $n \ll N$. Using extra informations, such as for instance $Q \geq 0$ in the case of LOCADIFF, allows to improve the efficiency and the accuracy of the inversion. It also proves useful to assume sparsity, that is to say that the changes are localized, such that $Q=0$ on a large majority of voxels. The assumption of sparsity increases the ratio $N / n$.

The reliability of the images produced by inversion is in most cases extremely important, as is their accuracy too. In two and three dimensions, the most common experimental cases, the resolution length $\varepsilon$ has to be chosen for an optimal quality of imaging balancing a low resolution for large values of $\varepsilon$ and inaccurate results for small values of $\varepsilon$. In the algorithm of Tarantola-Valette, 


\begin{tabular}{|c|c|c|c|}
\hline Technique & Input $(M)$ & Images $(Q)$ & Waves \\
\hline \hline CWI & relative delay & relative velocity & sound, elastic \\
\hline LOCADIFF & correlation loss & scattering & sound, elastic \\
\hline AV & $\log$ (intensity) & absorption & $\begin{array}{l}\text { sound, elastic, } \\
\text { light }\end{array}$ \\
\hline
\end{tabular}

TABLE II. Different imaging techniques using the average local time kernel. "Input" is the nature of the measurement, "Images" refers to the quantity one creates the image of. The column "Waves" indicates to which waves the technique can be applied. For elastic waves, this holds when waves are in the equipartition regime, which is a consequence of diffusion. The coda wave interferometry technique (CWI) uses time stretches on the wave field records to measure small velocity changes [5]. The LOCADIFF technique uses the correlation losses and produces images of the changes of scattering cross-section, that is to say the structural changes [7, 8]. The absorption variation (AV) has been described in Section III Note that as it is based on the intensity variations, the expected sensitivity of $\mathrm{AV}$ is smaller than for the other techniques.

the errors on the input measurements enter as a parameter of the inversion. The inversion provides an estimate of the errors on the results. One can therefore obtain, using the expressions for the fluctuations computed in the section $\mathrm{V}$ tune the resolution length for optimal results. The fluctuations - which expressions are provided by Equations (29), (32) and (34) - are due to the randomness of the medium and can therefore not be disposed of by repeating the measurements. To obtain independent, numerous enough input data, it is necessary to increase the number of measurement devices or use several disjoint time windows in the coda. The latter solution is however restricted by the quality of the recordings and the duration of the coda. As a conclusion from these remarks, it appears that the inverse problem based on a local time kernel is delicate and requires a good understanding of the different aspects of the problem.

\section{CONCLUSION}

In this article, I have introduced an elementary theory of local time for transport and its statistics. The average local time in infinite diffusive media and its fluctuations have been computed exactly for the dimensions one, two and three with a technique that is easily extended to higher dimensions in the appendix. Beyond the explicit expressions, it is important to note that the fluctuations explicitely depend on the resolution length, and diverge as the square root of the Green's function of the Laplace equation.

Wave propagation has a finite velocity which is not taken into account in the diffusion approximation. A more rigorous transport equation than the diffusion equation is the radiative transfer equation [10]. Solutions to this equation are known in dimensions one, two and three [16] and differ from the diffusion only at short times.
Clipping the integration domain of (12) is not correct because it does not account for the conservation of energy. The solutions to the radiative transfer contain ballistic terms that compensate this energy loss and preserve the conservation of energy. The short time discrepancy between diffusion and radiative transfer will change the average local time and its distribution close to the source and the receiver at distances of the order of the mean free path. A future improvement of this work is therefore turned to the radiative transfer solution of the wave equation in disordered medium. The solution to the twodimensional radiative transfer equation has been already used to numerically compute the local time [21].

Even though diffusion screens the medium's boundary conditions beyond a few mean free paths, these conditions are important in situations where the changes occur close to them. If the boundary is absorbing or reflecting, the average local time will be respectively reduced or increased. Simple boundary conditions, where the method of images applies, have been extensively discussed in Ref. [7].

Promising results [8, 9, 21], however, show that applications of local time to imaging offers new insights in physical sciences of complex media.

\section{ACKNOWLEDGMENTS}

This work was funded by grant number JC08-313906 from ANR and SMINGUE-UJF. The author whishes to thank Éric Larose and Thomas Planès for stimulating discussions.

\section{Appendix A: Laplace transform of time convolutions}

I note the multiple convolution of $n$ functions $f_{j}$

$h(t)=\int_{0}^{t} f_{1}\left(v_{1}\right) \mathrm{d} v_{1} \cdots \int_{0}^{t} f_{n}\left(v_{n}\right) \delta\left(t-v_{1}-\cdots-v_{n}\right) \mathrm{d} v_{n}$.

The integral can be performed from zero to infinity, since the Dirac distribution ensures that only values of $v_{j}$ between 0 and $t$ contribute to the result. The Laplace transform of the function $t \mapsto \delta\left(t-v_{1}-\cdots-v_{n}\right)$ is $\exp \left(-s v_{1}-\cdots-s v_{n}\right)$ therefore the Laplace transform of $h(t)$ is

$$
\hat{h}(s)=\int_{0}^{\infty} f\left(v_{1}\right) \mathrm{d} v_{1} \cdots \int_{0}^{\infty} f\left(v_{n}\right) \mathrm{d} v_{n} \mathrm{e}^{-s v_{1}-\cdots-s v_{n}} .
$$

The integrals are independent and each of them yields the Laplace transform of a function $f_{j}$. Therefore

$$
\hat{h}(s)=\hat{f}_{1}(s) \cdots \hat{f}_{n}(s) .
$$




\section{Appendix B: Computation of $I_{n}(x, y)$}

Let us compute the integrals

$$
I_{n}(\alpha, \beta)=\int_{0}^{1} \mathrm{e}^{-\alpha / u} \mathrm{e}^{-\beta /(1-u)} \frac{\mathrm{d} u}{u^{n+1}(1-u)^{n+1}}
$$

using the new variable $x=\frac{1-u}{u}$. We obtain

$$
I_{n}(\alpha, \beta)=\mathrm{e}^{-\alpha-\beta} \int_{0}^{\infty} \frac{(x+1)^{2 n}}{x^{n+1}} \mathrm{e}^{-\alpha x-\frac{\beta}{x}} \mathrm{~d} x .
$$

I note $J_{p}(\alpha, \beta)=\int_{0}^{\infty} \exp (-\alpha x-\beta / x) x^{-p-1} \mathrm{~d} x$. The value of $J_{p}$ is found in the Gradsteyn \& Ryzhik table [24, integral 3.471.9] :

$$
J_{-p}(\beta, \alpha)=J_{p}(\alpha, \beta)=2\left(\frac{\alpha}{\beta}\right)^{p / 2} K_{p}(2 \sqrt{\alpha \beta}),
$$

and we can express $I_{n}(\alpha, \beta)$ for $n>0$ as a sum

$$
\begin{aligned}
& I_{n}(\alpha, \beta)=\left(\begin{array}{c}
2 n \\
n
\end{array}\right) I_{0}(\alpha, \beta)+ \\
& 2 \mathrm{e}^{-\alpha-\beta} \sum_{p=1}^{n}\left(\begin{array}{c}
2 n \\
n-p
\end{array}\right) \frac{\alpha^{p}+\beta^{p}}{(\alpha \beta)^{p / 2}} K_{p}(2 \sqrt{\alpha \beta}) .
\end{aligned}
$$

\section{Appendix C: Computation of $\mu_{2}^{2}$}

The computation of $\mu_{2}^{2}(\boldsymbol{x}, t)$ is based on the integral

$$
M(\alpha, \beta, \epsilon)=\int_{0}^{1} \mathrm{~d} u \int_{0}^{1-u} \mathrm{~d} v \frac{\exp \left[-\frac{\epsilon}{u}-\frac{\alpha}{v}-\frac{\beta}{1-u-v}\right]}{u v(1-u-v)}
$$

We integrate $M$ thanks to the change of variable $x=$ $u /(1-u)$ after integrating over $v$ as in Appendix B

$$
M=2 \mathrm{e}^{-\epsilon-\alpha-\beta} \int_{0}^{\infty} \mathrm{e}^{-(\alpha+\beta) x-\epsilon / x} K_{0}(2 \sqrt{\alpha \beta}(1+x)) \frac{\mathrm{d} x}{x} .
$$

We use the Taylor expansion of $K_{0}$

$$
K_{0}(2 a(1+x))=\sum_{n, p \geq 0}\left(\begin{array}{l}
n \\
p
\end{array}\right) \frac{(-a)^{n} x^{n-1}}{n !} K_{n-2 p}(2 a)
$$

in the integral. Performing the integration over $x$ we get terms $J_{-n}(\alpha+\beta, \epsilon)$ defined by formula (B1). For $n \neq 0$ and $\epsilon \rightarrow 0$, we have $J_{-n}(\alpha+\beta, \epsilon) \simeq 2(n-1) !(\alpha+\beta)^{-n}$. The series rewrite

$$
\begin{aligned}
M & \simeq 4 \mathrm{e}^{-\alpha-\beta}\left\{K_{0}(2 \sqrt{\alpha \beta}) K_{0}(2 \sqrt{\epsilon(\alpha+\beta)})+\cdots\right. \\
& \left.+\sum_{n \geq 1, p \geq 0}\left(\begin{array}{c}
n-1 \\
p
\end{array}\right)\left(-\frac{\sqrt{\alpha \beta}}{\alpha+\beta}\right)^{n} K_{n-2 p}(2 \sqrt{\alpha \beta})\right\} .
\end{aligned}
$$

The leading term when $\epsilon \rightarrow 0$ is therefore logarithmic

$$
M(\alpha, \beta, \epsilon) \underset{\epsilon \rightarrow 0}{\simeq}-2 \mathrm{e}^{-\alpha-\beta} K_{0}(2 \sqrt{\alpha \beta}) \ln \epsilon .
$$

\section{Appendix D: Results in higher dimensions}

Although for direct applications in physics the higher dimensions are of little interest, some processes may be well described by a random walk in high dimensions. In this appendix, I give less detailed results concerning local times in dimensions higher than three.

\section{Average local time in higher even dimensions}

In a space of even dimension $d=2 n+2(n>0)$, we compute the time convolution (12) using the same technique as for $d=2$. We use the expression (B2) of $I_{n}(\alpha, \beta)$ and obtain

$$
\begin{aligned}
& L_{2 n+2}(\boldsymbol{x}, t)=\frac{t}{(4 \pi D t)^{n+1}} \exp \left[\frac{R^{2}-a^{2}-b^{2}}{4 D t}\right] \times \cdots \\
& \cdots \sum_{p=0}^{n}\left(2-\delta_{p}\right)\left(\begin{array}{c}
2 n \\
n-p
\end{array}\right)\left(\frac{a^{p}}{b^{p}}+\frac{b^{p}}{a^{p}}\right) K_{p}\left(\frac{a b}{2 D t}\right)
\end{aligned}
$$

where $\delta_{p}=1$ if $p=0$ and $\delta_{p}=0$ otherwise.

\section{Average local time in higher odd dimensions}

For the odd dimensions $d=2 n+3$, the we use the Laplace transform of $G_{d}$ in which the modified Bessel functions of the second kind are of half integral order and therefore have a simplified expression [20, eq. 9.7.2]. We obtain

$$
\hat{G}_{2 n+3}(a, s)=\mathrm{P}_{n}\left(a \sqrt{\frac{s}{D}}\right) \frac{\exp \left[-a \sqrt{\frac{s}{D}}\right]}{2(2 \pi)^{n+1} D a^{2 n+1}},
$$

where $\mathrm{P}_{n}$ is a unitary polynomial of degree $n$

$$
\mathrm{P}_{n}(x)=\sum_{p=0}^{n} \frac{(n+p) !}{2^{p} p !(n-p) !} x^{n-p} .
$$

The product $\hat{G}_{2 n+3}(a, s) \hat{G}_{2 n+3}(b, s)$ contains therefore the product of two polynomials $\mathrm{P}_{n}$, which is a unitary polynomial of degree $2 n$. Let us express it in the basis of the $\mathrm{P}_{k}$ polynomials in $(a+b) x$ :

$$
\begin{aligned}
& \mathrm{P}_{n}(a x) \mathrm{P}_{n}(b x)= \\
& \quad \frac{a^{n} b^{n}}{(a+b)^{2 n}} \sum_{k=0}^{n} \mathrm{Q}_{k}^{n}\left(\frac{a}{b}+\frac{b}{a}\right) \mathrm{P}_{2 n-k}((a+b) x),
\end{aligned}
$$

where $\mathrm{Q}_{k}^{n}$ is a polynomial of degree $\leqslant k$ (see table 【II). Although a general decomposition formula on this basis would require to sum up to $k=2 n$, the sum is limited to $n$ because the term of order $k$ gives after Laplace inversion a terms $G_{4 n-2 k+3}(a+b, t) / G_{2 n+3}(R, t)$ which decreases as $t^{k-n}$. For $t \rightarrow \infty$, the local time must remain finite and therefore one has $k \leq n$. 


\begin{tabular}{|c||c|c|c|c|c|}
\hline$k$ & 0 & 1 & 2 & 3 & 4 \\
\hline $\mathrm{Q}_{k}^{1}(X)$ & 1 & $X-1$ & & & \\
$\mathrm{Q}_{k}^{2}(X)$ & 1 & $3 X-4$ & $3\left(X^{2}-X-1\right)$ & & \\
$\mathrm{Q}_{k}^{3}(X)$ & 1 & $6 X-9$ & $3\left(5 X^{2}-8 X-1\right)$ & $15\left(X^{3}-X^{2}-2 X+1\right)$ & \\
$\mathrm{Q}_{k}^{4}(X)$ & 1 & $10 X-16$ & $9\left(5 X^{2}-10 X+2\right)$ & $15\left(7 X^{3}-12 X^{2}-6 X+8\right)$ & $105(X-1)\left(X^{3}-3 X-1\right)$ \\
\hline
\end{tabular}

TABLE III. The $\mathrm{Q}_{k}^{n}$ polynomials for $n \leqslant 4$. The value of $\mathrm{Q}_{0}^{n}$ is always equal to 1 .

The average local time kernel in arbitrary odd dimensions is therefore given by

$$
\begin{aligned}
& L_{2 n+3}(\boldsymbol{x}, t)=\frac{a+b}{2 D(2 \pi a b)^{n+1}} \exp \left[\frac{R^{2}-(a+b)^{2}}{4 D t}\right] \cdots \\
& \cdots \times \sum_{k=0}^{n}\left(\frac{(a+b)^{2}}{2 D t}\right)^{n-k} \mathrm{Q}_{k}^{n}\left(\frac{a}{b}+\frac{b}{a}\right) .
\end{aligned}
$$

\section{Fluctuations of local time in higher dimensions}

In higher dimensions as in dimensions 1,2 and 3 , the moment of order 2 is proportionnal to the singu-

[1] D. J. Pine, D. A. Weitz, P. M. Chaikin, and E. Herbolzheimer, Phys. Rev. Lett. 60, 1134 (1988).

[2] M. L. Cowan, I. P. Jones, J. H. Page, and D. A. Weitz, Phys. Rev. E 65, 066605 (2002).

[3] É. Larose and S. Hall, J. Acoust. Soc. Am. 125, 1853 (2008).

[4] E. Niederleithinger and C. Wunderlich, AIP Conf. Proc. 1511, 390 (2013).

[5] C. Pacheco and R. Snieder, J. Acoust. Soc. Am. 118, 1300 (2005).

[6] J. Battaglia, J.-P. Métaxian, and E. Garaebiti, Geophys. Res. Lett. 39, L11309 (2012).

[7] V. Rossetto, L. Margerin, T. Planès, and É. Larose, J. Appl. Phys. 109, 034903 (2011).

[8] E. Larose, T. Planès, V. Rossetto, and L. Margerin, Appl. Phys. Lett. 96, 204101 (2010).

[9] A. Obermann, É. Larose, T. Planès, and M. Campillo, "Imaging pre- and co-eruptive structural changes of a volcano with ambient seismic noise," submitted (2013).

[10] S. Chandrasekar, Radiative transfer (Dover, 1960).

[11] D. Revuz and M. Yor, Continuous martingales and Brownian motion (Springer, New York, 1991).

[12] H. F. Trotter, Ill. J. Math. 2, 425 (1958). larity $g_{d}(\varepsilon)=1 / \mathcal{S}_{d} \varepsilon^{d-2}\left(\mathcal{S}_{d}\right.$ is the surface of the $d$ dimensional sphere).

$$
\frac{\Lambda_{d}(\boldsymbol{x}, t)}{L_{d}(\boldsymbol{x}, t)} \simeq \frac{1}{\sqrt{D \mathcal{S}_{d} L_{d}(\boldsymbol{x}, t) \varepsilon^{d-2} / 2}}
$$

[13] P. Lévy, Processus stochastiques et mouvement brownien (Gauthier-Villars, Paris, 1965).

[14] T. Shang and L. Gao, Scientia Sinica B 31, 1503 (1988).

[15] H. Sato, Geo. Phys. J. Int. 117, 487 (1993).

[16] J. C. J. Paasschens, Phys. Rev. E 56, 1135 (1997).

[17] B. Gittenberger and G. Louchard, J. App. Math. Stoch. An. 13, 125 (2000).

[18] J. Pitman, in Séminaire de probabilités XXXIII, edited by A. J., M. Émery, M. Ledoux, and M. Yor (Springer, 1999) pp. 388-394.

[19] C. Gardiner, Handbook of stochastic methods, 4th ed. (Springer, 2006).

[20] M. Abramowitz and I. A. Stegun, Handbook of mathematical functions, 10th ed. (Dover, 1972).

[21] A. Obermann, T. Planès, É. Larose, C. Sens-Schönfelder, and M. Campillo, Geophys. J. Int. 194, 372 (2013).

[22] A. Tarantola and B. Valette, J. Geophys. 50, 159 (1982).

[23] E. Candès, J. Romberg, and T. Tao, IEEE Trans. Inf. Theory 52, 489 (2006).

[24] I. S. Gradsteyn and I. M. Ryzhik, Table of integrals, series and products, sixth edition ed. (Academic Press, London, 2000). 